\title{
Adaptive Pushing of Learning Resources in Fragmented English Reading
}

\author{
Jianmin Zhang ${ }^{\mathrm{a}, \mathrm{b}}$, Min Xie ${ }^{\mathrm{a}, \mathrm{b}, *}$, and Bin Wen ${ }^{\mathrm{a}, \mathrm{b}}$ \\ ${ }^{a}$ School of Information, Yunnan Normal University, Kunming, 650500, China \\ ${ }^{b}$ Key Laboratory of Educational Information for Nationalities, Ministry of Education, Kunming, 650500, China
}

\begin{abstract}
With the development of social economy, science and technology, and the popularity of mobile Internet, human society has gradually entered the information age. The rapid growth and disorderly distribution of information have posed challenges to English learners who conduct independent and individualized learning anytime and anywhere. Reading plays an important role in language learning. Traditionally, it is difficult for the English reading method based on chapters to meet the fragmented learning needs of learners who are often in a mobile environment. Through the literature research, this paper designed the five-dimensional feature model of learners and the three-dimensional feature model of English reading resources in the fragmented learning environment. Combined with the machine learning algorithm ID3, the decision tree classification model of English fragmented reading was constructed to push resources, adapt to the individual needs of the learners, and then specifically enhance the different aspects of the learner's reading ability, thereby improving the pass rate of the College English Test-Band Four.
\end{abstract}

Keywords: adaptive push; ID3 algorithm; learner model; English reading; fragmented learning

(Submitted on October 21, 2018; Revised on November 22, 2018; Accepted on December 25, 2018)

(C) 2019 Totem Publisher, Inc. All rights reserved.

\section{Introduction}

With the rapid development of information technology, mobile terminal equipment is becoming more and more intelligent, and people's reading habits and reading methods have also changed. The method of traditional paper reading can no longer meet the needs of learners, and fragmented reading is becoming increasingly popular with college students. College students have many courses and high academic pressure. By using this new reading method, students can accumulate English knowledge bit by bit in fragmented time, not only in the limited time and space of English classes. Students can learn English autonomously according to their own needs and acceptance ability to improve their interest and learning effect in English learning. The application of fragmented learning in the field of education has gradually been emphasized, and its influence on English learning has become more and more prominent.

Compared to holistic reading, fragmented reading refers to an incomplete and intermittent reading mode through new media such as mobile phones, e-books, and mobile networks [1]. With the help of mobile terminals such as smart phones and mobile networks, learners can receive a large amount of information anytime and anywhere. In the process of learning, information and knowledge seem to be readily available, and reading seems very easy [2].

However, when students use common network resources to learn, English reading resources are abundant, diversified, and highly shared, which brings convenience to people but also brings the problem of overload of network information. Learners cannot get the part of information that is particularly valuable to them quickly when they face a huge amount of information. Reading is a complex cognitive process, but the existing English reading App makes learning plans in pushing learning resources just based on learning tasks, learning objectives, or learning needs, without fully considering the changes of learners in the dynamic learning process. As more and more people use App to learn, the problem of learners' learning adaptability in this environment is becoming increasingly prominent. It is difficult for learners to achieve real online self-

* Corresponding author.

E-mail address: 294611018@qq.com 
learning, and it is more difficult to obtain adaptive learning resources [3]. How can we help students quickly and accurately find the learning resources that suit their individual needs in the process of learning to meet the individual needs of learners in the mobile learning process? Personalized recommendation can solve the problem of information overload. It enables information consumers to quickly get the content they need, while also allowing the information provider to provide targeted information to the user in a targeted manner and push the information that the target user wants most. This method is actually a recommended method. Therefore, in the English fragmented reading system with adaptive function, it is necessary to construct a learner model and a resource model that are suitable for this learning scenario, After that, we need to select a suitable machine learning algorithm, construct a model for a large number of user learning behaviors, extract push rules, predict information that is adapted to the user's needs, and recommend the content to the user [4].

In summary, this paper collects the feature data of the learner in the learning process according to the individual needs of the learner and makes it suitable for data mining through preprocessing in the mobile and fragmented learning environment. Then, the data is randomly divided into training set and test set, and the resource push decision tree is established by using the ID3 algorithm to find the rules of resource push, so as to realize the personalized push of CET-4 reading resources in the mobile learning environment to meet the needs of fragmented reading.

\section{Adaptive Pushing of Learning Resources in English Fragmented Reading}

\subsection{Adaptive Learning System}

Adaptive learning is actually a study of individual differences. It is a highly personalized learning process that allows the learning environment, learning content, and learning activities to adapt to each individual's different characteristics [5]. It can be said that adaptive learning is a kind of learning that varies from person to person and is rich in personalized learning.

The Adaptive Learning System (ALS) is an active learning system that is based on the "learner-centered" educational philosophy and proposes individual differences for current learners, (such as age, professional background, interest preferences, and cognitive level). It dynamically provides a system for personalized learning support services. The adaptive learning system was first introduced in the field of foreign intelligent teaching systems and adaptive hypermedia systems. In recent years, it has become a hot spot in the field of E-Learning distance education [6-7]. The personalized push mechanism is the core component of the adaptive learning system, and its main function is to effectively match and reorganize learning resources to meet the personalized learning needs of current users [8]. With the help of the adaptive learning system, learners can monitor their own learning process and choose the learning content and learning strategy that suits them best according to their actual needs [9]. In the adaptive learning situation, learning is not a process of passively accepting knowledge, but a process of actively discovering knowledge. Adaptive learning generally occurs in a distance learning environment, which refers to the process in which learners acquire knowledge and acquire abilities through their original knowledge and experience interacting with the distance learning system. In this process, students can self-organize the learning process by developing and implementing learning plans, controlling the entire learning process, and self-assessing the learning [10].

At present, the English learning App on the mobile phone is becoming more and more popular with the younger generation. It is more common to use the fragmented learning method for English reading and learning. The goal of this type of App is to provide learners with personalized learning, that is, learners' learning interests, behaviors in the learning process, learning history and learning level, and other learner characteristics. Adaptive learning resources provide a learning path that meets the learner's individual needs.

\subsection{Adaptive Push Model of Learning Resources in English Fragmented Reading}

In order to achieve adaptive push of learning resources in the English fragmented reading environment, it is first necessary to determine learner characteristics and resource characteristics, use the machine learning algorithm to automatically find the push strategy, and then push the appropriate resources to the appropriate learners at the appropriate time. On the basis of literature review, it is found that in the fragmented learning environment, learner models can be established according to learners' reading ability, cognitive style, learning target, learning situations, learning results, and other information in fourlevel English reading. Then, extract the feature information of English reading resources and establish the connection between learner model and learning resources. Thereafter, based on the feature information of the learner, the sequence of knowledge points to be learned is generated. Based on this sequence, the push strategy is determined, the learning path is planned, and the learning resources are pushed.

In this paper, an adaptive push model of learning resources in English fragmented reading is shown in Figure 1. 


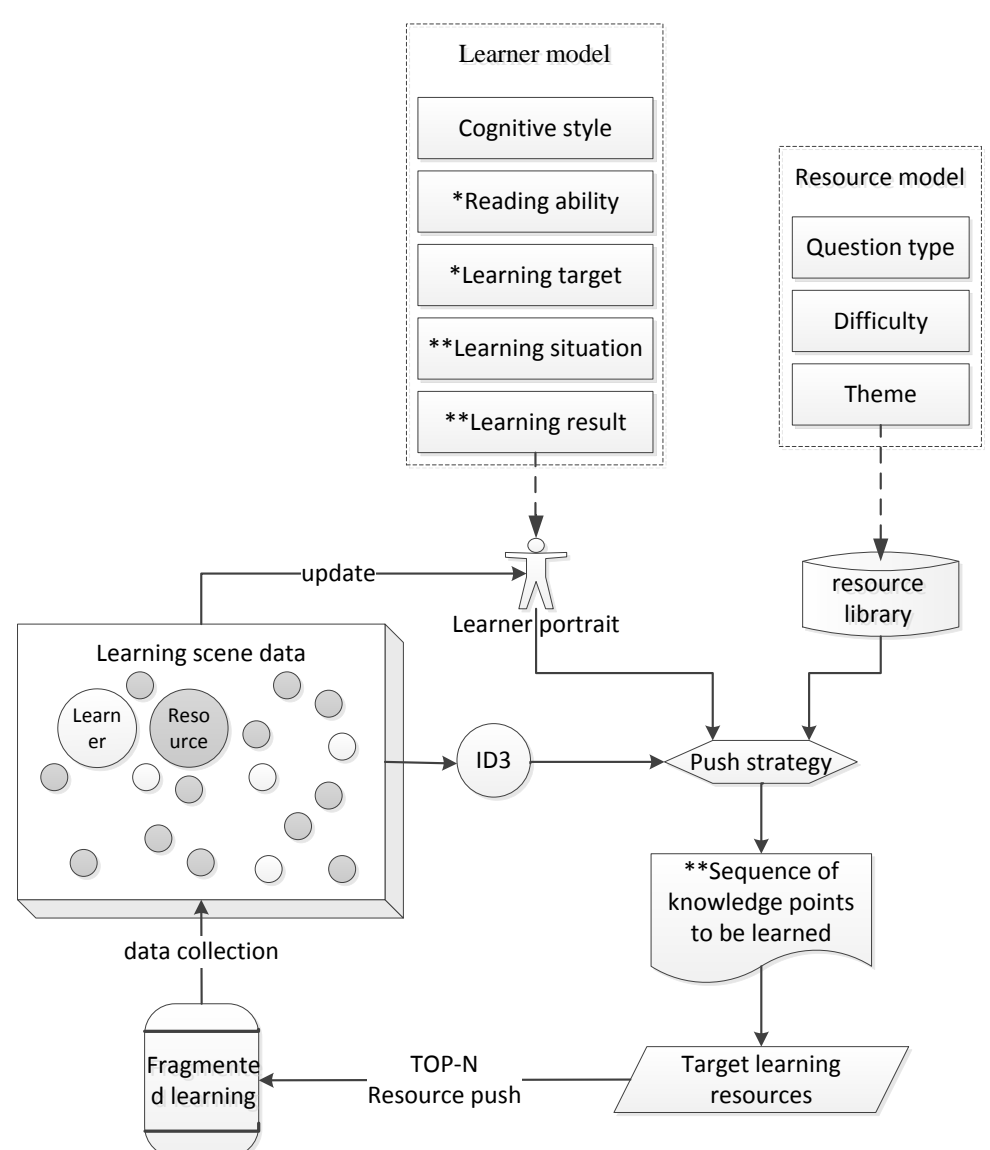

Figure 1. Adaptive learning resources pushing model in English fragmented reading

Note:

(1) In the learning scene, the white circle represents learners, and the gray circle represents the learning resources fragmented.

(2) “*” represents the relative stability of data. Items without "*” are more stable, items with “*” take a longer time to update, and items with “**” update the fastest.

\subsubsection{Learner Model}

In the fragmented learning environment, a learner model is constructed according to the learners' reading ability, cognitive style, learning target, learning situation, and learning results in the process of English reading. The learner model shows that in order to realize the self-adaptive push of resources, the system needs to understand what level the learners are at, what goals they want to achieve, where and how to learn, and how the effect is based on resource push learning. Among them, due to the particularity of changing places in mobile learning, the influence of the information obtained by learning the situation on the fragmented learning effect is more important than the influence of learning in other environments.

\section{(1) English reading ability}

The assessment of learners' reading ability $(\mathrm{Ra})$ is based on the CET-4 syllabus. It is mainly based on the Chinese English Reading Ability Rating Scale [11] and the seven factors mentioned in the article A Study on the Structure and Development of College Students' English Reading Ability [12]. Develop an assessment scale for English reading ability. The Chinese English Reading Ability Rating Scale has nine levels. Among them, the first and second levels correspond roughly to the primary school level, the third level corresponds to the junior middle school level, the fourth level corresponds to the senior high school level, the fifth and sixth levels correspond to the university level, the seventh level corresponds to the English professional level, and the eighth and ninth levels correspond to the high-end foreign language talent level. This study does not involve primary school, junior high school, or high-end talents, so it selects grades 4-7. 
The seven factors in the structure of English reading ability include: 1) external decoding of word meaning, internal decoding of words; 2) mastering the grammatical structure; 3 ) internal and external decoding of the meaning of the sentence; 4) an overall understanding of articles of different genres; 5) language ability; 6) the surface understanding of discourse, including logical meaning, time and body, and different influences of lexical chain on textual cohesion; 7) an understanding of the coherence of discourse.

Extract the relevant topics related to the above seven English reading ability structures in grades 4-7 of the English Reading Competency Rating Scale in the Chinese English Reading Ability Rating Scale. They are adapted to the English reading ability assessment scale for adaptive learning of learning resources in English fragmented reading, which is recorded as $\{B 4, B 5, B 6, B 7\}$.

\section{(2) Cognitive style}

The so-called Cognitive Style (Cs) is the individual difference in the organization and processing of information and experience. It is the habitual attitude and style that individuals often adopt in the process of perception, memory, and thinking [13]. Reading is a complex cognitive activity. Different cognitive styles will inevitably lead learners to show different reading tendencies and reading habits during the reading process. In English reading, Field Independence (FI) and Field Dependence (FD) cognitive style students have significant differences in reading interest, thinking patterns, environment, and learning strategies.

Field-independent students prefer natural science-related articles to independent reading; they are good at focusing on the content related to the purpose of reading first and grasping the main points of the article by analytical methods. They are often independent of the surrounding environment in perception and are not susceptible to external factors. They have strong autonomy, like to study and think independently, are good at discovering problems, and can reorganize the content taught and integrate it into their own knowledge.

Field-dependent students are more interested in reading content related to people and society and like group learning; they are easily influenced by the comprehensive thinking mode and are accustomed to reading articles from the recognition and refinement of words and sentences. Their perception of things tends to take external reference as the basis of information processing, and they are more susceptible to the influence and interference of the surrounding environment. However, they are willing to learn in a collective situation. In the collective, field-dependent learners are more submissive, can get along well with others, and like to help others. They are also susceptible to others' hints and need more feedback, and their objectives and courses should be explained in detail. They prefer non-analytical, general, and holistic perceptual learning, and their learning is less active.

\section{(3) Learning target}

This study is aimed at undergraduate students who are studying English at the fourth level and are preparing to apply for the exam. The main learning target (Lt) of this group is passing the College English Test-4 (CET-4). The phased goal is defined as the amount of words and the correct rate of reading comprehension achieved by the learner in a certain stage.

\section{(4) Learning situation}

In the process of mobile learning, the learning situation (Ls) has a relatively large impact on the effect of mobile learning. The uncertainty of the learning situation has become one of the factors that hinder learners from completing personalized adaptive learning in the mobile environment. This study mainly considers three aspects of learning situations:

Firstly, the geographical location of learning occurs, which is mainly measured by the influence of the noise of geographical location on learners' learning status and is expressed by the decibel value of the geographical environment. The sound of the learning situation is divided into three grades: noisy ( $>60 \mathrm{~dB})$, which has a greater impact on learner's learning or poor learning effect; normal $(40-60 \mathrm{~dB})$, which is suitable for learners to learn normally; and quiet (0-40dB), which is the best learning state [14].

The second is the best time for learning efficiency, so as to determine the difficulty of pushing resources. The system first records the current time of the user's learning and then comprehensively obtains the time period during which the learner is highly efficient in the day to judge the learner's best learning time. 
The third is continuous learning time, based on the continuous time of the questions available to learners, to determine the size of the push resources. A reading material usually contains 4-5 questions, and the learner takes 5-10 minutes to complete it. In fragmented reading, such a reading material needs to be split into fragments related to the problem so that the learner can complete the learning within 1-2 minutes of waiting for the bus or waiting in line.

\section{(5) Learning result}

In this study, learning result $(\mathrm{Re})$ is mainly feedback through the correct rate of the problem of the learner in the complex learning situation. If it is correct, it is recorded as: True. The error is recorded as: False.

\subsubsection{Feature Model of English Reading Resources}

The type, theme, and difficulty of reading materials have a greater impact on learners. Different types of questions essentially examine different ways of thinking. Based on different growth environments, learners have different familiarity with reading materials on different subjects, and the results of the questions vary greatly. However, subjects with different degrees of difficulty require learners to be at different levels of learning. Understanding these differences in reading materials, the system can push resources more specifically for learners. This study extracts the feature information of English reading resources from the three-dimensional degree of topic type, subject matter, and difficulty level of reading resources and establishes a three-dimensional reading resource feature model.

Among them, the question types (Qt) are divided into detailed questions and non-detail questions. Detailed questions include Factual detail (Fd), Inference problem (Ip), and Semantic comprehension problem (Scp). Non-detailed questions include the Main idea (Mi) and the Attitude of view problem (Avp) [15]. Theme (Th) is divided into social sciences (Sc) and natural sciences (Ns). Difficulty (Di) grades are divided into Difficult (Dif) and Easy (Ea). Therefore, the feature model of English reading resources is recorded as qquestion type, theme, difficulty\}.

With the five-dimensional learner model (cognitive style, reading ability, learning target, learning situation, and learning result) and three-dimensional resource feature model (question type, theme, difficulty), we can use the machine learning algorithm ID3 to analyze the data, find the corresponding relationship between learners and learning resources, and construct learners' individual, so as to precipitate the rule. It is convenient to recommend the learning resource for the learner in the subsequent learning.

\section{Application of ID3 Algorithm in Adaptive Pushing of English Fragmented Reading Resources}

In 1966, Hunt first applied the decision tree method in the concept learning system CLS (Care Life of Science) [16], which laid the foundation of the decision tree algorithm. In 1975, Quinlan proposed a decision tree algorithm [17]. Decision tree is a classification algorithm based on data structure [18-19], whose core is the ID3 algorithm. The algorithm chooses the attribute with the highest information gain as the test attribute of the current node, which minimizes the amount of information needed for data classification in the result partition, and embodies the minimum randomness of partition. For a given classification problem, the decision tree construction process is top-down, divide and conquer. Starting from the root node, the given data samples are tested, and the data samples are divided into several sub-sample sets according to the test results. Each sub-sample set constitutes a new sub-sample. The tree building process is iterated until the given termination condition is satisfied [20]. In the final decision tree, each non-leaf node corresponds to a non-category attribute. The leaves indicate that the attribute is a value. The path from root to leaf node corresponds to a rule [21], and a decision tree corresponds to a set of disjunctive expression rules. The ID3 algorithm has strong learning ability and is suitable for largescale data processing. The principle of the algorithm is simple and easy to understand. It can generate easy-to-understand classification rules, and the classification speed of the algorithm is fast [22].

\subsection{The Basic Idea of ID3 Algorithm}

\subsubsection{Calculate the Information Entropy of Sample Classification}

Assuming that for the $X$ training set, the target attribute $C$ of $X$ has $m$ possible class label values and the frequency of occurrence of $C_{i}$ in all samples in training set $X$ is $(i=1,2,3, \cdots, m)$, then the information entropy contained in the training set $X$ is defined as: 


$$
\operatorname{Entropy}(X)=\operatorname{Entropy}\left(P_{1}, P_{2}, \cdots, P_{m}\right)=-\sum_{i=1}^{m} P_{i} \log _{2} P_{i}
$$

Among them, $p_{i}$ is the probability that any sample belongs to $C_{i}$ and is estimated by $X_{i} / X$. Because the information is encoded in binary system, the logarithm is based on 2 .

\subsubsection{Calculate the Information Entropy of Other Sets Divided by Attribute A}

Assuming that attribute $A$ has $k$ different values and $X$ is divided into $k$ sample subsets $\left\{X_{1}, X_{2}, \cdots, X_{k}\right\}$, then the information entropy of the sample subset divided by attribute $A$ is as follows:

$$
\operatorname{Entropy~}_{A}(X)=\sum_{i=1}^{k} \frac{\left|X_{i}\right|}{|X|} \operatorname{Entropy}\left(X_{i}\right)
$$

Among them, $\left|X_{i}\right|(i=1,2, \cdots, k)$ is the number of samples contained in the subset of $X_{i}$ and $|X|$ is the number of samples contained in the sample set $X$.

\subsubsection{Calculate the Information Gain of Attributes}

Assuming that the sample data set before division is $X$ and the sample set $X$ is divided by the attribute $A$, then the information gain $\operatorname{Gain}(X, A)$ of the attribute $X$ is divided into the entropy of the sample set $X$ minus the entropy of the sample subset after the $X$ is divided by the attribute $A$ :

$$
\operatorname{Gain}(X, A)=\operatorname{Entropy}(X)-\text { Entropy }_{A}(X)
$$

The information gain of each attribute is calculated according to Equations (1) to (3). The larger the information gain, the purer the subset of attribute $A$, which is more conducive to classification. Select the attribute with the greatest information gain as the test attribute of the sample set $X$, construct a node, create a branch for each value of the attribute, continue to partition, and finally construct a decision tree.

\subsection{ID3 Algorithm for Adaptive Push in English Fragmented Reading}

\subsubsection{Calculate the Information Entropy of Sample Classification}

As can be seen from the foregoing, the original classification category is judged by "the quality of the recommended resources", that is, $C=\{Y E S, N O\}$. According to Equation (1), the category attribute is $C$, which can be obtained:

$$
\begin{aligned}
& \text { Entropy }(X)=\operatorname{Entropy}\left(P_{1}, P_{2}\right)=-\sum_{i=1}^{2} P_{i} \log _{2} P_{i} \\
& =-P_{Y E S} \log _{2} P_{Y E S}-P_{N O} \log _{2} P_{N O}=-\frac{X_{Y E S}}{X} \log _{2} \frac{X_{Y E S}}{X}-\frac{X_{N O}}{X} \log _{2} \frac{X_{N O}}{X}
\end{aligned}
$$

From this, we can get the information entropy of the first classification.

\subsubsection{Calculate the Information Entropies of Other Sets Divided by Candidate Attributes}

As can be seen from the foregoing, eight attributes should be considered in adaptive resource push, which are question type, theme, difficulty, reading ability, cognitive style, learning target, learning situation, and learning result, expressed as Th, $Q t$, $D i, R a, C s, L t, L s$, and $R e$ respectively. Each attribute has a different range of values: attribute $T h$ has 2 kinds of values $\{S c, N s\}, Q t$ has 5 values $\{F d, I p, S c p, M i, A v p\}, D i$ has 2 values $\{D i f, E a\}, R a$ has 5 values $\{B 4, B 5, B 6, B 7\}, C s$ has 2 values $\{F i, F d\}, L t$ has 2 values $\{y o u, w u\}, L s$ has 3 values $\{$ noisy, normal, quiet $\}$, and $R e$ has 2 values $\{$ True, False $\}$. The conditional entropy of each attribute is calculated separately, and the information entropy of other attributes is calculated under the classification category condition. 
Taking attribute $T h$ as an example, the information entropy of attribute $T h$ is as follows:

There are $n$ samples, in which the attribute $T h$ is $S c$ with $n_{c}$ bars and the attribute $T h$ is $N s$ with $n_{s}$ bars. The "recommended good" category in $S c$ is yes with $n_{m}$ bars and no with $n_{n}$ bars. In Ns, the category "recommended good or bad" is yes with $n_{i}$ bars and no with $n_{j}$ bars, and then according to Equation (2):

$$
\begin{aligned}
\text { Entropy }_{T h}(X)= & \sum_{i=1}^{k} \frac{\left|X_{i}\right|}{|X|} \operatorname{Entropy}\left(X_{i}\right) \\
= & \frac{\left|X_{S c}\right|}{|X|} \operatorname{Entropy}\left(X_{S c}\right)+\frac{\left|X_{N s}\right|}{|X|} \operatorname{Entropy}\left(X_{N s}\right) \\
& =\frac{n_{c}}{n}\left[-\frac{n_{m}}{n_{c}} \log _{2}\left(\frac{n_{m}}{n_{c}}\right)-\frac{n_{n}}{n_{c}} \log _{2}\left(\frac{n_{n}}{n_{c}}\right)\right] \\
& +\frac{n_{s}}{n}\left[-\frac{n_{i}}{n_{s}} \log _{2}\left(\frac{n_{i}}{n_{s}}\right)-\frac{n_{j}}{n_{s}} \log _{2}\left(\frac{n_{j}}{n_{s}}\right)\right]
\end{aligned}
$$

Similarly, we can calculate the conditional entropy of the $Q t, D i, R a, C s, L t, L s$, and $R e$ attributes under the recommended quality category.

\subsubsection{Calculate the Information Gain of Attributes, and then According to Equation (3):}

$$
\operatorname{Gain}(T h)=\operatorname{Entropy}(X)-\operatorname{Entropy}_{T h}(X)
$$

From this, the information gain of $T h$ can be calculated.

Similarly, the $Q t, D i, R a, C s, L t, L s$, and $R e$ attributes are calculated. Select the maximum information gain value as the node, create a branch for each value of the attribute, continue to divide recursively in this way, and finally construct a decision tree for fragmented reading resource push.

\subsection{Improved ID3 Algorithm}

The basic idea of resource adaptive push is to automatically adjust the recommendation strategy according to the change of the learner's attribute, so as to adapt to the change of the learner and push the most suitable learning resources. However, not every dimension in the learner model has the same update speed. If only part of the learner attribute participates each time the push policy is updated, it can greatly reduce the amount of computation, improve the system response speed, and thus improve the user experience.

In the learner model, the learner's cognitive style is the habitual behavior pattern exhibited by the individual in the cognitive process. It is a process variable rather than a content variable, with stability across time and consistency across contexts. Therefore, it can be considered that the individual's cognitive style is fixed and unchanged for a relatively long time. The learner's English reading ability refers to the ability to comprehend, analyze, summarize, guess, and judge the article in reading practice and after reading. After a period of study, the reading ability will be adjusted, and it will change after a long period of study. The learner's learning target refers to the change in behaviors and abilities of the learner through learning, especially the changes in the behaviors, skills, and emotions that the learner should achieve after completing a certain stage or unit of learning. The learning target in English reading mainly refers to the increase in vocabulary and the correct rate of problem-solving achieved by learners in a certain stage. Therefore, the learning target will change in a certain period of time. The learning situation is the physical environment in which the learner is learning, and it will change with the change of the learning environment. The learning result is the result of completing a unit of learning, so it changes with the learner's learning.

Based on the above characteristics of the learner model, the algorithm is improved on the basis of the original ID3 algorithm. Before the next login, the user automatically extracts the last saved decision tree of the user and in turn determines whether the root node and the branch nodes have changed; if not, it then retains the last decision tree. If the part changes, the unchanged part retains the previous decision tree node and reconstructs the decision tree from the changed part. 
If it changes completely, it reconstructs the whole decision tree.

For a given training set sample, the test attribute set is attribute -list $=\left\{A_{1}, A_{2}, \cdots, A_{n}\right\}$, the category attribute is $C$, and $A_{i}$ is any attribute of the test attribute set. The specific steps of the ID3 algorithm are as follows:

S1 Create node Node;

S2 To determine whether the sample is the same type of output;

$\mathbf{S 3}$ If so, the node becomes a leaf and uses this type of mark;

S4 To determine whether the attribute list is empty;

S5 If yes, return the node as a leaf, and mark the category as the category with the largest number of output category instances in the sample;

S6 Select the attributes with the highest information gain, which best classify samples;

S7 This property becomes the branching attribute of the node. (Note: In this algorithm, all attributes are discrete, and attributes with continuous values must be discretized);

S8 Traversing each known value of the branching attribute;

S9 A branch with a condition of test-attribute $=a_{i}$ is generated by the node Node;

S10 Let $S_{i}$ be the set of test-attribute $=a_{i}$ samples in samples;

S11 If $S_{i}$ is empty;

S12 Add a leaf and record it as the most common class in the samples;

S13 If $\mathrm{Si}$ is not empty, plus a node returned by ID3_Decision_Tree $\left(\mathrm{S}_{\mathrm{i}}\right.$, attribute-list, test-attribute), once an attribute appears on a node, it is not necessary to consider the attribute on any descendants of the node.

Note: recursive partitioning steps only stop when one of the following conditions is established:

- All samples of a given node belong to the same class (S2 and S3).

- There are no remaining attributes that can be used to further partition the sample (S4). In this case, a majority vote (S5). This involves converting a given node into a leaf, marking it with the class in which most of the samples are located.

- Branch test-attribute $=\mathrm{a}_{\mathrm{i}}$, no sample (S1). In this case, create a leaf (S12) with most of the classes in the samples.

The pseudo code is as follows:

Function ID3_Decision_Tree (samples, attribute-list)

\{

1. Create a node: Node;

2. IF samples is in the same category $\mathbf{C}$. then

3. return Node is a node with class $\mathbf{C}$ tags;

4. IF attribute-list is Null then

5. return Node is the leaf node and is labelled as the most common class in samples;

6. select the highest gain attribute test-attribute from attribute-list;

7. mark the attribute attribute-list as node: Node;

8. $\quad$ known values for each test-attribute $\mathbf{A} \mathbf{i}$;

9. according to the condition test-attribute $=\mathrm{a}_{\mathrm{i}}$, the node Node is divided into a new branch;

10. let $\mathbf{S i}$ be the set of test-attribute $=a_{i}$ samples in samples;

11. IF $\mathbf{S}_{\mathrm{i}}$ is Null THEN

12. add a leaf node and mark it as the most common class in samples;

ELSE

13. add a node returned by ID3_Decision_Tree ( $\mathrm{S}_{\mathrm{i}}$, attribute-list);

\section{Experiment and Result}

\subsection{Collecting Data and Building Decision Tree}

According to the principle of convenient sampling, 12 students of a certain class who are preparing for CET-4 are selected as the initial sample to collect 120 pieces of fragmented reading data. The ID3 algorithm is used to push learning resources close to learners' personalized needs based on learner models. From the collected data, $75 \%$ of the data was randomly selected as the training data set to construct the corresponding binary tree classification model; the remaining $25 \%$ was used 
as the test data set to evaluate the accuracy of the created binary tree model. When the correct rate of evaluation is above $95 \%$, the model is considered to be effective. According to the foregoing, the initial classification category is judged by "the quality of recommended resources". That is, $C=\{Y E S, N O\}$. The decision tree established based on the test set of the obtained samples is shown in Figure 2.

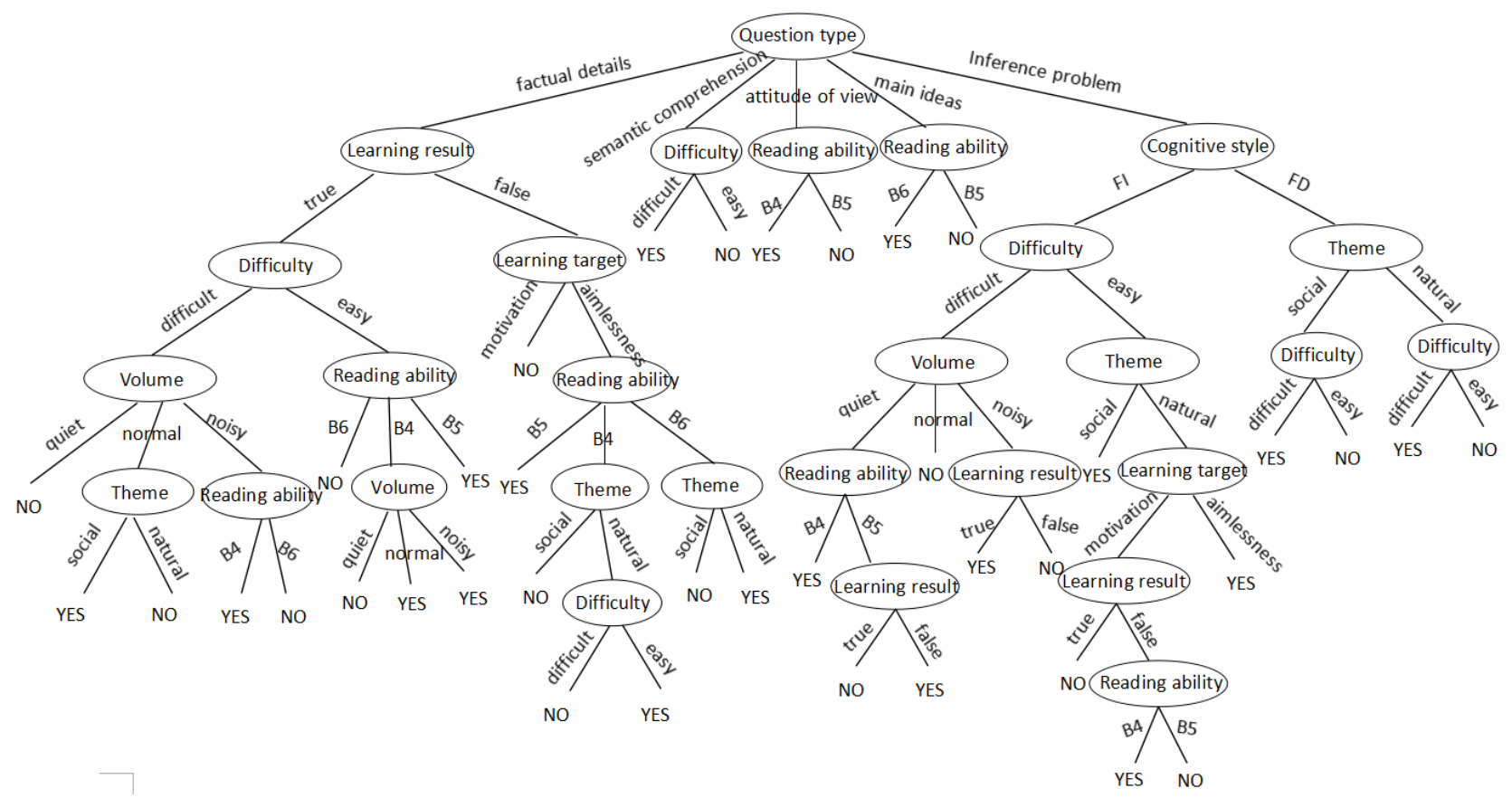

Figure 2. The decision tree of resources pushing in English fragmented reading

The classification rules of test data are similar to those of decision trees established by sample data. Mainly through the resource type, the learner's learning effect, the difficulty of resources, the learner's reading ability, and the cognitive style to establish nodes, the following branches only differ in the volume of resources and the volume of fragmentation learning situations. Therefore, it is believed that the initial creation of fragmented reading resources is effective in pushing decision trees. The accuracy of this experiment is about $71.4 \%$. Because of the small sample size, the decision tree established has more branches and is quite different from the decision tree created on the training set, but the main nodes are similar. Although the accuracy rate is less than $95 \%$, it can still be considered that the Resource Recommendation decision tree is effective to a certain extent. In order to improve the accuracy of recommendation, further data collection is needed, such as the development of a dedicated App for a wider range of trials to verify and improve the recommendation algorithm.

\subsection{Discussion}

In English fragmented reading based on resource push, through the analysis of the decision tree created by the ID3 algorithm, the results show that the main factors affecting the effect of resource push are the question type of resources, the learning effect of the learner, the difficulty of resources, the learner's reading ability, and the cognitive style. In the decision tree, it also reflects the learning objectives and the subject matter of resources (humanities and social sciences, natural sciences). The volume decibel of the fragmented learning situation does not directly affect the learner's learning effect, but it is related to the branch in which the attribute is located.

For the question type, the point of view and the subject matter are influenced by the reading ability of the learners. The problem of semantic comprehension is only related to the difficulty of the topic. For the factual details and reasoning judgment questions in detail, different resources are recommended to different learners in different learning situations, and the learning effect is different.

It can be seen that when the learner conducts four-level English fragmented reading, the strategy of resource push should mainly start from the learner's reading ability and cognitive style and combine the types of reading resources. At the same time, in the process of mobile learning, learners should consciously conduct targeted and staged learning on resources of different difficulty levels. Only in this way can we improve the reading comprehension ability of CET-4 and the passing rate of CET-4. 


\section{Conclusions}

In the fragmented learning context, this paper, through the collection and analysis of learners' reading ability, cognitive style, learning objectives, learning situations, and learning effects in the learning process, establishes a five-dimensional learner feature model combined with the types of reading resources, theme, and difficulty of the three-dimensional feature model. The machine learning algorithm ID3 is used to establish the connection between the learner model and the reading resources.

When constructing a decision tree with the ID3 algorithm, choose the attribute with the largest information entropy as the root node. Every time a root node is determined, the maximum information entropy must be calculated again, so that the new root node must be determined again. It must be recursion calculated many times. It provides a framework for the Learning Resource Recommendation System in English fragmented learning. Using Apps with the self-adaptive push function of learning resources in English reading, learners can make full use of fragmented time to study, which is beneficial to improve their reading comprehension ability and the passing rate of CET-4.

In future works, the ID3 algorithm will be studied to improve and optimize the learning resource recommendation algorithm in English fragmented reading, to reduce the time consumption of the classifier, to improve the accuracy of recommendation, and to optimize the classification process in order to further improve the adaptability of the algorithm.

\section{Acknowledgments}

The research is supported by the National Nature Science Fund Project (No. 61562093, 61661051), Key Project of Applied Basic Research Program of Yunnan Province (No. 2016FA024), Program for Innovative Research Team (in Science and Technology) in University of Yunnan Province, and Starting Foundation for Doctoral Research of Yunnan Normal University (No. 2017ZB013).

\section{References}

1. C. B. Wang, X. P. Li, F. N. Zhao, et al., "Fragmentation Learning Research in the Age of Big Data," E-Education Research, Vol. 36, No. 10, pp. 26-30, 2015

2. G. K. Yuan, "Fragmented Reading Can't Replace Traditional Reading," Yunnan Daily: Night Talk in the Book World, Vol. 12, June 2010

3. X. H. Gao, "Research on Network-based Adaptive Learning System," Shanghai Normal University, 2003

4. L. Zhu, "Research and Application of User Recommendation Method based on Classification Algorithm," Beijing University of Posts and Telecommunications, 2017

5. Y. Ruan, "Adaptive Learning: Teaching Students in Accordance with the Support of Information Technology_—Interviewing Professor Wang Lu of Capital Normal University," Primary and Secondary School Information Technology Education, No. 11, pp. 4-6+11, 2006

6. L. J. Mou, “Adaptability Research of Networked Learning System,” E-Education Research, No. 5, pp. 48-52, 2009

7. X. K. Zhao, X. D. Xu, and S. R. Long, "Research on Personalized Recommendation Service of Adaptive Learning System in B/S Mode," Distance Education in China, No. 10, pp. 71-78, 2015

8. X. K. Zhao and L. Cen, "Research on Personalized Learning Path Recommendation for Adaptive Learning System based on User Demand," China Education Info, No. 21, pp. 28-31, 2016

9. X. Q. Liu, "Research on the Creation of Domain Ontology in Adaptive Learning System based on Semantic Web," Northeast Normal University, 2008

10. S. Q. Yu and K. K. He, "Research on Adaptive Learning System based on Internet Network," in Proceedings of the 3rd Global Chinese Computer Application Conference, 1999

11. "Chinese English Reading Ability Rating Scale was Officially Released by the Ministry of Education and the National Language Committee," (http://www.china-language.gov.cn/yw/gjywxx/201804/t20180418_29983.html, accessed May 15, 2018)

12. P. P. Bao and J. F. Zhang, "Research on the Structure and Development of College Students' English Reading Ability," Journal of Shaoxing University, Vol. 24, No. 2, pp. 116-120, 2004

13. Q. Y. Yao, "An Experimental Study of the Relationship Between English Reading Strategies and Cognitive Styles," Journal of Huzhou University, Vol. 29, No. 6, pp. 125-128, 2007

14. H. Y. Yu, "Design of Personalized Learning System based on Situational Perception on WeChat Public Platform," Yunnan Normal University, 2017

15. "National College English Test Band 4 and 6 (Revised 2016)," (http://www.cet.edu.cn/file_2016_1. pdf, accessed August 2018)

16. B. W. Porter, E. R. Baress, and R. Holte, "Concept Learning and Heuristic Classification in Weak Theory Domains," Artificial Intelligence, Vol. 45, pp. 229-263, 1989

17. J. R. Quinlan, "Induction of Decision Trees," Machine Learning, No. 1, pp. 81-106, 1986

18. L. A. Brealow and D. W. Aha, "A Survey. Navy Center for Applied Research in Artificial Intelligence," Naval Blesearch Lab, Washington DC 20375, 1996

19. L. A. Brealow and D. W. Aha, "Simplifying Decision Trees: A Survey," The Knowledge Engineering Review, Vol. 2, pp. 1-40, 1997 
20. W. Y. Xiang, "Application Research of ID3 Algorithm in English Score Analysis," Journal of Liuzhou Vocational and Technical College, Vol. 11, No. 2, pp. 31-34, 2011

21. M. Fan, "Application of ID3 Algorithm in Analysis of Students' Achievements," Software Guide, Vol. 9, No. 12, pp. 65-66, 2010

22. A. H. Huang and X. T. Chen, "Improvement of Decision Tree ID3 Algorithm," Computer Engineering and Science, Vol. 31, No. 6, pp. 109-111, 2009

Jianmin Zhang is a postgraduate student in the Key Laboratory of the Ministry of Education for Ethnic Minorities at Yunnan Normal University, majoring in digital learning environment and technology.

Min Xie is an associate professor, doctor, and Master's tutor in the Information School at Yunnan Normal University, studying digital learning environments and technology and ethnic education informatization.

Bin Wen is an associate professor, doctor, and Master's tutor in the Information School at Yunnan Normal University, studying ethnic education informatization. 